\title{
Quiste epidermoide intradiploico infratentorial gigante
}

\author{
F. Alberione; F. Caire; D. Fischer-Lokou; M. Gueye y J.J. Moreau
}

Servicio de Neurocirugía. Centro Hospitalario Universitario de Limoges. Hospital Universitario Dupuytren. Universidad de Limoges.

\section{Resumen}

Los quistes epidermoides son lesiones congénitas benignas, de lento crecimiento, que representan el $1 \%$ de todos los tumores intracraneales. En un $25 \%$ tienen una localización intradiploica y excepcionalmente infratentorial.

Presentamos un caso clínico poco frecuente de un quiste epidermoide intradiploico gigante infratentorial. El paciente consultó por una diplopia y un síndrome cerebeloso. La tomografía computarizada y la resonancia magnética mostraron una lesión gigante lítica extradural de la fosa posterior de $5,2 \mathrm{~cm} \times 3,8 \mathrm{~cm}$, con realce periférico con el contraste. Utilizamos un abordaje suboccipital retrosigmoideo para realizar una exéresis completa tumoral y una resección incompleta capsular, debido a la adherencia de ésta al seno lateral.

La evolución posoperatoria fue favorable.

Realizamos una revisión bibliográfica de la literatura y discusión de nuestro caso.

PALABRAS CLAVE: Quiste epidermoide. Intradiploico. Infratentorial.

\section{Giant intradiploic infratentorial epidermoid cyst}

\section{Summary}

Epidermoid cysts are benign, uncommon lesions (1\% of all intracranial tumors). Their localization is intradiploic in $\mathbf{2 5 \%}$ of cases, and exceptionally subtentorial.

We report here a rare case of giant intradiploic infratentorial epidermoid cyst. A 74-year old patient presented with recent diplopia and sindrome cerebellar. CT scan and MR imaging revealed a giant osteolytic extradural lesion of the posterior fossa $(5,2 \mathrm{~cm} \times 3,8 \mathrm{~cm})$ with a small area of peripheral enhancement after contrast injection. Retrosigmoid suboccipital craniectomy

Recibido: 19-01-07. Aceptado 30-03-07 allowed a satisfactory removal of the tumor, followed by an acrylic cranioplasty. The outcome was good. Neuropathological examination confirmed an epidermoid cyst.

We review the literature and discuss our case.

KEY WORD: Epidermoid cyst. Intradiploic. Infratentorial.

\section{Introducción}

Los quistes epidermoides (QE) intracraneales son lesiones congénitas de bajo grado de crecimiento e histológi camente benignas. Representan alrededor del 1\% de todos los tumores intracraneales. Es conocido como "el tumor perlado" por sus características macroscópicas de color nacarado. Sus localizaciones más frecuentes son a nivel del ángulo pontocerebeloso, región supraselar y cuadrigeminal ${ }^{2,15,21}$.

Los quistes epidermoides intradiploicos son aproximadamente el $25 \%$ de todos los $\mathrm{QE}^{3}$. La ubicación en la fosa posterior es poco frecuente.

Presentamos el caso clínico de un paciente con diagnóstico de QE intradiploico infratentorial gigante.

\section{Caso clínico}

Paciente de 74 años, con antecedentes de miocardiopatía isquémica, que consultó por una diplopia de instalación brusca y un síndrome cerebeloso derecho. En el examen neurológico presentaba una discreta dismetría derecha y una ataxia cerebelosa. El examen oftalmológico fue normal.

La tomografía computarizada mostraba una lesión expansiva extradural de la fosa posterior, hipodensa, de 5,2 $\mathrm{cm} \times 3,8 \mathrm{~cm}$, con calcificaciones pericapsulares, con erosión ósea occipital y con realce periférico con el contraste (Fig 1).

En la resonancia magnética esta lesión se presentaba

Abreviaturas. QE: quiste epidermoideo. TAC: tomografía axial computarizada. RMN: resonancia magnética nuclear. 


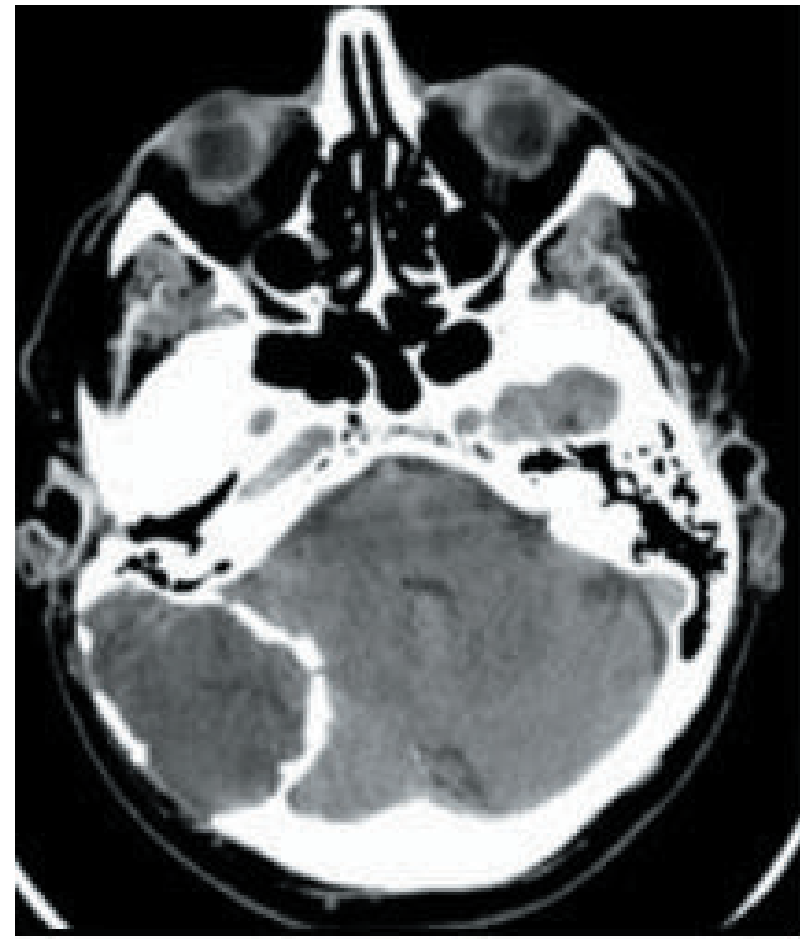

Figura 1. TAC craneal sin contraste. Se aprecia lesión expansiva osteolítica hipodensa extradural con calcificaciones pericapsulares de la fosa posterior.

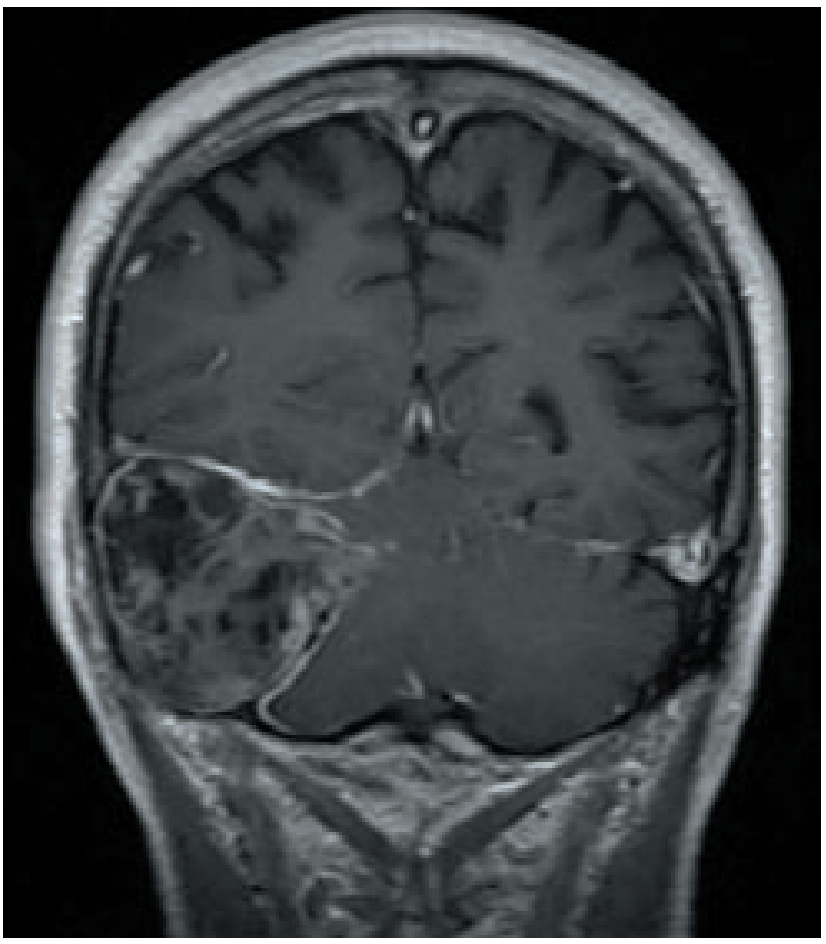

Figura 3. RMN craneal corte coronal T1, en donde se evidencia el realce periférico con Gadolinio y la poca diferenciación de la cápsula a medial.

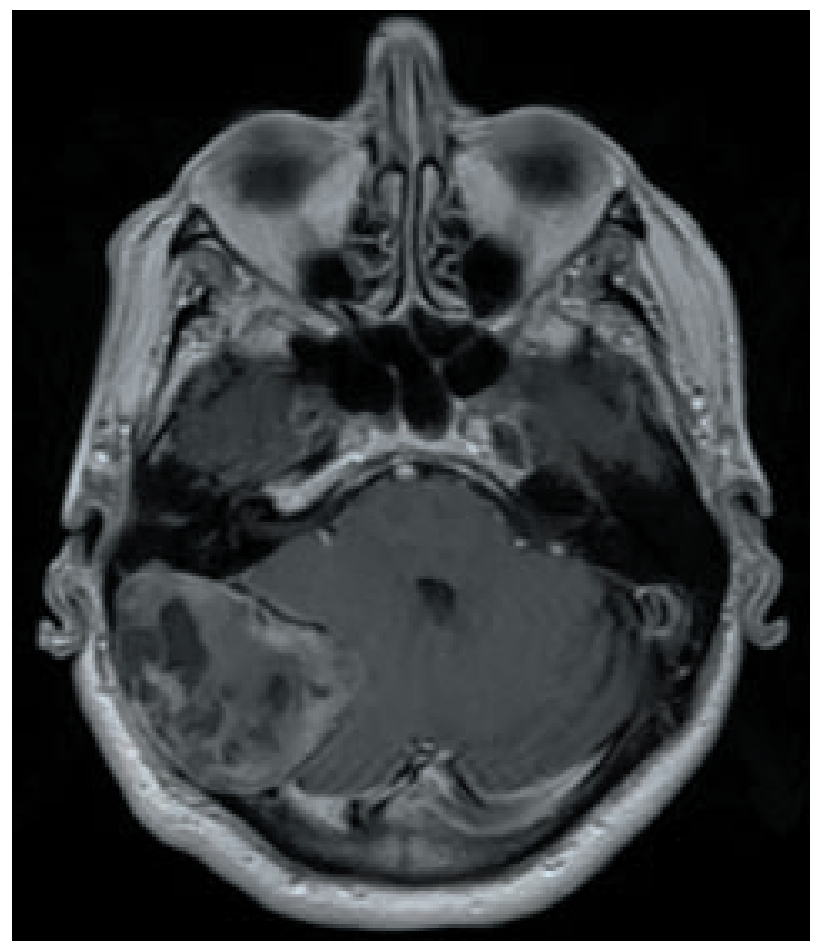

Figura 2. RMN craneal corte axial muestra una lesión heterogénea hipointensa en T1.

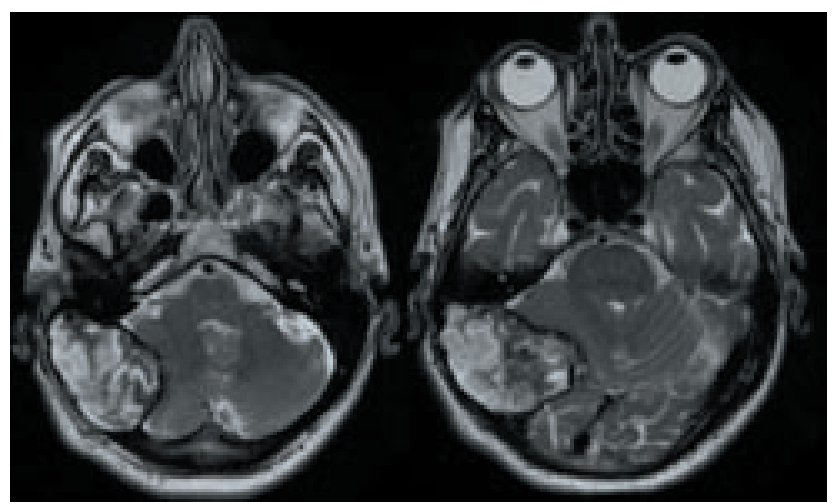

Figura 4. RMN craneal T2 axial se observa lesión hiperintensa heterogenea.

como heterogénea hipointensa con realce periférico con el gadolinio en T1 (Figs 2-3) e hiperintensa en T2 (Fig 4).

Realizamos una intervención quirúrgica en posición sentada y abordaje suboccipital retrosigmoideo derecho. Macroscópicamente observamos un tumor extradural heterogéneo, friable, de color blanco-verdoso. Se realizó una resección "tumoral" completa. A nivel de la parte más interna de éste, la diferenciación entre la cápsula y la duramadre no era clara, por lo que procedimos a una exéresis de la duramadre de dos centímetros aproximadamente y posterior plástica con duramadre sintética. También fue dificultosa la disección de la cápsula a nivel del 


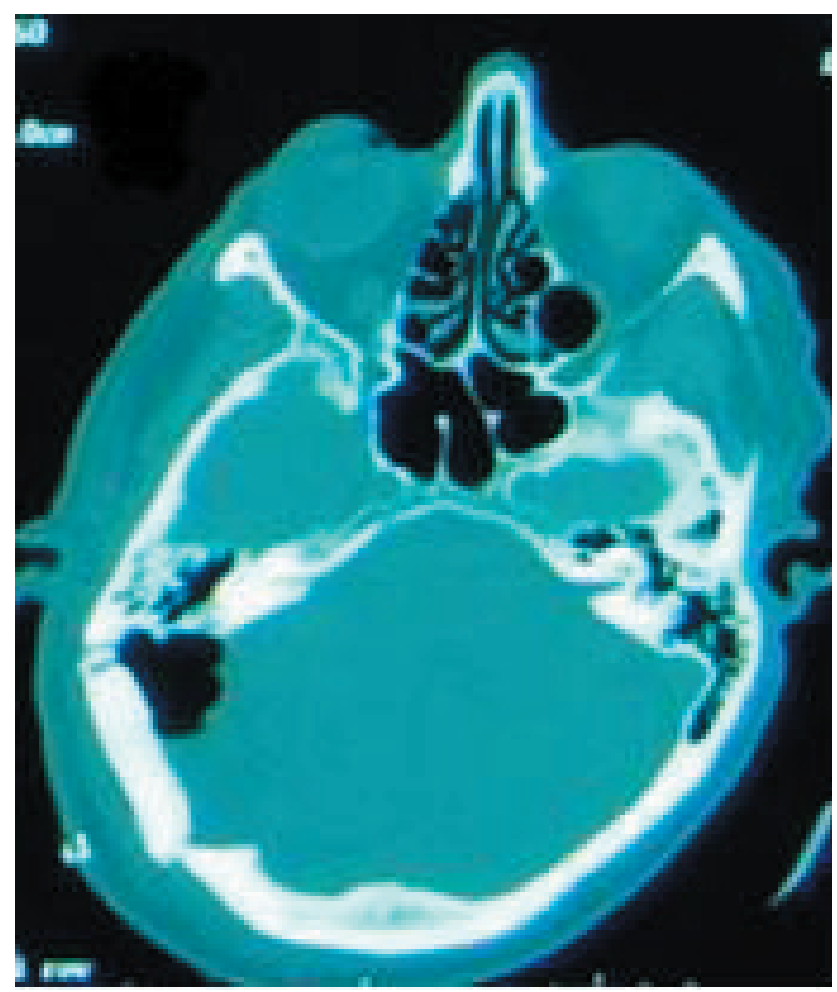

Figura 5. TAC craneal de control posoperatorio. Ventana ósea: se aprecia la craneoplastia y un pequeño neumoéncefalo.

seno lateral, por lo que se procedió a una exéresis incompleta a este nivel. Finalmente, realizamos una craneoplastia con cemento quirúrgico.

El examen anatomopatológico microscópico mostró una lesión quística constituida por una pared de epitelio malpingiano queratinizado y tejido óseo. El contenido estaba constituido por numerosas láminas de queratina. El diagnóstico histopatológico fue de quiste epidermoideo intradiploico.

La evolución posoperatoria fue favorable sin ninguna complicación. La tomografía computarizada de control muestra un pequeño neumoencéfalo (Fig. 5).

\section{Discusión}

Los QE son lesiones benignas que se originan de restos de tejidos epitelial ectodérmicos que quedan en el sistema nervioso central al cerrarse el tubo neural entre la tercera y quinta semana de gestación ${ }^{12}$. La inclusión de células epidérmicas a nivel óseo es el origen de la forma intradiploica ${ }^{2}$. También se pueden producir en forma adquirida a través de punciones lumbares o intraóseas.

La velocidad de crecimiento es lenta, de tipo linear (similar a la tasa de crecimiento de la piel), contrariamente a la mayoría de los tumores que tienen un crecimiento exponencial.

La localización más frecuente de los QE es intradural. Se presentan a nivel del ángulo pontocerebeloso, de la región paraselar y cuadrigeminal ${ }^{2,15,21}$. También se han descrito casos en otras localizaciones menos frecuentes, como en la Cisura de Silvio, región quiasmática, a nivel ventricular ${ }^{6,10,17}$ intrapontino ${ }^{12}$, talámicos ${ }^{8}$ o del cuerpo calloso.

Aproximadamente el 25\% de los QE son intradiploicos ${ }^{3}$. Las localizaciones más habituales son en el hueso frontal y parietal. Los QE intradiploicos a nivel occipital son poco frecuentes. En la revisión bibliográfica encontramos descritos a nivel occipital 7 casos de 37 en la serie de Arana et $\mathrm{al}^{2}, 2$ de 4 en la serie de Bikmaz et $\mathrm{al}^{4}$, y un caso presentado por Bhora et $\mathrm{al}^{3}$, Guridi et $\mathrm{al}^{11}$, Kalgutkar et $\mathrm{al}^{14}$ y Maiuri et $\mathrm{al}^{16}$.

Nuestro caso coincide con las características atípicas de los 8 casos de Arana et $\mathrm{al}^{2}$, y el caso de Bhora et $\mathrm{al}^{3}$ en cuanto al tamaño mayor de $5 \mathrm{~cm}$ y la irregularidad de los bordes lesional. Otras series descritas de QE gigantes son los 9 casos de Jaiswal et a $1^{13}$ y 3 casos de Ciapetta et $\mathrm{al}^{9}$.

Estos tumores pueden ocurrir a cualquier edad, pero hay un ligero predominio entre la segunda y quinta década ${ }^{2,3,4,18}$. Nuestro caso coincide con el trabajo de Bhora et col. ${ }^{3}$ de un paciente mayor de 70 años. No hay diferencia de incidencia con respecto al sexo.

Clínicamente se pueden manifestar por alteración de pares craneales ${ }^{15}$ o síndrome cerebeloso a nivel infratentorial. Los QE supratentoriales se presentan por cefaleas, crisis comiciales, déficit motores o sensitivos y/o hidrocefalia. A nivel orbitario y frontal pueden ocasionar exoftalmia y/o trastornos visuales ${ }^{5}$. También se pueden diagnosticar como lesión tumoral (QE de la calota craneal) o en forma fortuita. Pueden desarrollar gran tamaño hasta ocasionar sintomatología clínica ${ }^{4,9}$.

En la tomografía computarizada aparecen como lesiones hipo o isodensas, generalmente sin captación de contraste, salvo en algunos $\operatorname{casos}^{3,21}$, como el nuestro, donde hay un mayor riesgo de malignización. La variedad intradiploica se aprecia como una lesión lítica con erosión ósea. Pueden observarse calcificaciones. Megdiche-Bazarbacha et $\mathrm{col}^{18}$ han descrito un raro caso de un QE hiperdenso espontáneamente en la fosa posterior.

En la resonancia magnética se observan como lesiones hipointensas en T1 e hiperintensas en T2, como en nuestro caso. La secuencia de difusión y FLAIR se pueden utilizar para realizar diagnóstico diferencial de quiste aracnoideo y en el control de residuo tumoral en las exéresis parcia$\operatorname{les}^{17,22}$.

Entre los diagnósticos diferenciales podemos citar a los colesteatomas (antecedentes de infección otológicas) ${ }^{1}$, los quistes dermoides (poseen contenidos dérmicos y se localizan frecuentemente en la línea mediana), el granuloma eosinófilo (pacientes jóvenes y bordes óseos no escleróticos) y 
las metástasis de la calota craneana (bordes irregulares).

El tratamiento de los QE es la exéresis completa del tumor y de su cápsula en la medida de lo posible, implicando esto un buen pronóstico ${ }^{1,9,11}$. En las lesiones intradurales las adherencias a estructuras vasculo-nerviosas no permiten, a veces, realizar una resección completa. La cápsula debe extirparse totalmente; sin embargo, como en nuestro caso, la adherencia al seno lateral imposibilitó la resección completa. El residuo capsular es un predisponente a la recidiva, aunque el crecimiento es habitualmente lento.

La tasa de resección completa de los QE intradurales varía entre un $18-95 \%$.

Dentro de las complicaciones posoperatorias cabe mencionar la meningitis aséptica y la infección.

La transformación maligna de los QE a carcinoma de células escamosas es rara, pero esta descrita en la literatura $^{7}$. Su tratamiento es discutido, y a pesar de la radioterapia y/o la quimioterapia el pronóstico no es favorable ${ }^{19,20}$.

\section{Bibliografía}

1. Altschuler, E., Jungreis, C., Sekhar, L., Janetta, P., Sheptak, P.: Operative treatment of intracranial epidemoid cyst and cholesterol granulomas: report of 21 cases. Neurosurgery 1990; 26: 606-614.

2. Arana, E., Latorre, F., Revert, A., Menor, F., Riesgo, P., Liaño, F., Diaz, C.: Intradiploic epidermoid cysts. Neuroradiology 1996; 38: 306-311.

3. Bhora, A., Emery, E., Khouri, S., Hitier, M., Derlon, J.: Kyste épidermoïde extradural geant de la fosse postérieure. Neurochirurgie 2005; 51: 599-603.

4. Bikmaz, K., Cosar, M., Bek, S., Gokduman, C.,Arslan, M., Iplikcioglu, A.: Intradiploic epidermoid cysts of the skull: a report of four cases. Clin Neurol Neurosurg 2005; 107: 262-267.

5. Blanco, G., Esteban, R., Galarreta, D., Saornil, M.: Orbital intradiploic giant epidermoid cyst. Arch Ophthalmol 2001; 119: 771-773.

6. Bougeard, R., Mahla, K., Roche, P., Hallacq, P., Vallée, B., Fisher, G.: Kyste epidermoïdes des ventricules latéraux. Neurochirurgie 1999; 45: 316-320.

7. Bretschneidert, T., Dorenbeck, U., Strotzer, M., Roth, M., Rummele, P., Buettiner, R.: Squamous cell carcinoma arising in an intradiploic epidermoid cyst. Neuroradiology 1999; 41: $570-572$.

8. Chandler, W., Farhat, S., Pauli, F.: Intrathalamic epidermoid tumor. Case report. J Neurosurg 1975; 43: 614-617.

9. Ciapetta, P., Artico, M., Salvati, M., Raco, A., Gagliardi, F.: Intradiploic epidermoid cyst of the skull. Report of ten cases and review of the literature. Acta Neurochir 1990; 102: 33-37.
10. Gelabert-González, M., García-Allut, A., GonzálezGarcia, J., Martínez-Rubio, R.: Quiste epidermoide de tercer ventrículo. Neurocirugía 2002; 13: 389-392.

11. Guridi, J., Ollier, J., Aguliera, F.: Giant intradiploic epidermoid tumour of the occipital bone: case report. Neurosurgery 1990; 27: 978-981.

12. Izquierdo, J., Sala, J.: Intrapontine epidermoid cyst. Neurocirugía 1991; 2: 117-120.

13. Jaiswal, A., Mahapatra, A.: Giant intradiploic epidermoid cysts of the skull. A report of eight cases. Br J Neurosurg 2000; 14: 225-228.

14. Kalgutkar, A., Kini, S., Jambhekar, N., Das, S.: Intradiploic primary epithelial inclusion cyst of the skull. Ann Diagn Pathol 2006; 10: 20-23.

15. Kobata, H., Kondo, A., Iwasaki, K.: Cerebellopontine angle epidermoids presenting with cranial nerve hiperactive dysfunction: pathogenesis and long-term surgical results in 30 patients. Neurosurgery 2002, 50: 276-286.

16. Maiuri, F., Del Basso de Caro, M., D Acunzi, G., Tortora, F., Esposito, F.: Giant intradiploic epidermoid cyst of the occipital bone. Zentralbl Neurochir 2004; 65: 32-35.

17. Marin, H., Vargas, M., Grebici-Guessoum, M., et col.: Kystes epidermoïdes du quatrième ventricule. A propos de quatre cas. J Neuroradiol 2002; 29: 146-152.

18. Megdiche-Bazarbacha, H., Nagi, S., Douira, W., Sebai, R., Belghith, L., Touibi, S.: Kyste épidermoide intracranien spontanément hyperdense. J Neuroradiol 2004; 31: 150-152.

19. Michael, L., Moss, T., Madhu, T., Coakham, H.: Malignant transformation of posterior fossa epidermoid cyst. Br J Neurosurg 2005; 19: 505-510.

20. Parikhs, S., Milosovic, M., Wong, C., Laperriere, N.: Recurrent intracranial epidermoid cyst treated with radiotherapy. J. Neurooncol 1995; 24: 293-297.

21. Talacchi, A., Sala, F., Alessandrini, F., Turazzi, S., Briccolo, A.: Assessment and surgical management of posterior fossa epidermoid tumours: report of 28 cases. Neurosurgery $1998 ; 42: 242-252$.

22. Tsuruda, J., Chew, W., Moseley, M., Norman, D.: Diffusion-weighted MR imaging of the brain: value of differentiating between extraaxial cysts and epidermoid tumors. AJNR Am J Neuroradiol 1190: 11; 925-931.

Alberione, F.; Caire, F.; Fischer-Lokou, D.; Gueye, M.; Moreau, J.J.: Quiste epidermoide intradiploico infratentorial gigante. Neurocirugía 2007; 18: 423-426.

Dirección postal: Dr. Français Caire. Service de Neurochirugie. Centre Hospitalaire Universitaire de Limoges. Hôspital Universitaire Dupuytren. 2, avenue Martin-Luther-King. 87042. Limoges Cedex. 